\title{
Computed tomography measurement of the bone matrix of vertebral pedicle and its clinical significance
}

\author{
X. Li' ${ }^{1}$ X. Wang ${ }^{1}$, S. Gao ${ }^{1}$, H. En ${ }^{1}$, Y. Zhang ${ }^{2}$, H. Wang ${ }^{1}$, Y. Cai' ${ }^{1}$ Z. Wang ${ }^{1}$, \\ Z. Li', C. Zhang ${ }^{3}$, J. Ma' ${ }^{1}$, S. Zhang ${ }^{1}$ \\ 'Department of Anatomy, Inner Mongolia Medical University, Inner Mongolia, P.R. China \\ 2Department of CT Center, The Second Affiliated Hospital of Inner Mongolia Medical University, Inner Mongolia, P.R. China \\ ${ }^{3}$ The Third Clinical College of Inner Mongolia Medical University, Inner Mongolia, P.R. China
}

[Received: 22 November 2018; Accepted: 10 January 2019]

\begin{abstract}
Background: To provide the anatomic basis for the clinical application of the transpedicular screw fixation.

Materials and methods: Thirty spine $\left(C_{2}-L_{5}\right)$ specimens were used. The width of the pedicle cortex and width of the pedicle medullary cavity (WPC and WPMC), and the height of the pedicle cortex and height of the pedicle medullary cavity (HPC and HPMC) were measured at the isthmus of the pedicle using computed tomography (CT) scanning.

Results: Width of the pedicle medullary cavity changed in a three-dovetailed-saddle shape with four peaks and three valleys, namely $C_{2}$ (high), $C_{4 \cdot 5}$ (low), $T_{2}$ (high), $T_{4}$ (the lowest), $T_{12}$ (high), $L_{1}$ (low) and $L_{5}$ (the highest). HPMC of the cervical pedicle changed in a saddle shape, gradually increasing from $C_{5}-L_{5}$.WPC, WPMC, HPC and HPMC showed a regular change, respectively. In each segment, the superior border of the pedicle cortex had a nearly consistent thickness to the interior border within an identical pedicle, while the pedicle cortex thickness radio of the medial and lateral border was nearly 3:1 among the cervical pedicles, 2:1 among thoracic pedicles, and 1:1 among lumbar pedicles.

Conclusions: Both HPMC and WPMC are the dominant factors for the choice of screw diameter, but HPMC should also be considered in $\mathrm{C}_{2}-T_{1}$ pedicles, especially $C_{6}$ and $C_{7}$. Additionally, the screw for $C_{3-6}$ or $T_{4-6}$ pedicles should be about $3.0 \mathrm{~mm}$ in diameter. (Folia Morphol 2019; 78, 3: 476-480)
\end{abstract}

Key words: vertebral pedicle, bony cortex, medullary cavity, screw diameter

\section{INTRODUCTION}

Posterior transpedicular screw fixation of the spine is widely used in clinical practice as an important fixation method for the spine disorder. However, due to the large morphological changes in different segments of the vertebra, especially the internal morphological structure of the pedicle, improper selection of screw specifications and poor screw placement can lead to spinal cord injury and other serious complications [4].

Choosing the proper screw diameter, which is determined by width of the pedicle medullary cavity (WPMC) and/or the height of the pedicle medullary

Address for correspondence: Z. Li and S. Zhang, Department of Anatomy, Basic Medical College, Inner Mongolia Medical University, Jinshan Economic Development Zone, Huhhot 010110, Inner Mongolia, P.R. China, tel/fax:+8604716657553, e-mail: 798242742@qq.com Xiaohe Li and Yunfeng Zhang are co-first authors or contributed equally to this work. 


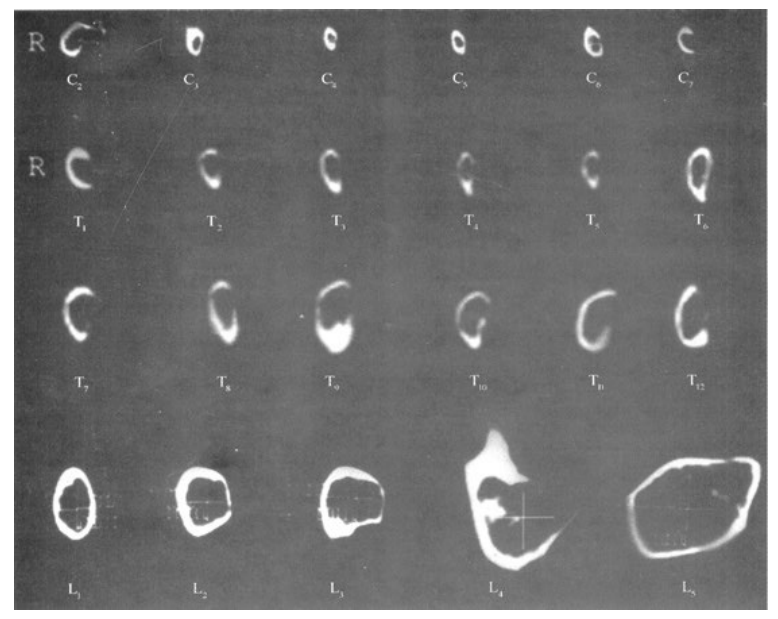

Figure 1. Vertical computed tomography image of the pedicles isthmus.

cavity (HPMC) is one of the critical factors in transpedicular screw fixation. However, there are only a few reports concerning the measurement of the medullary cavity in the pedicle and the bony cortex thickness of each pedicle. Ninomiya et al. [4] measured the cortex thickness of both medial and lateral borders of vertebral pedicles in $\mathrm{L}_{3-5}$ of 5 spines. Wu et al. [7] measured WPMC of pedicles in $L_{1-5}$ of 100 spines using computed tomography (CT) scanning. Li et al. [3] conducted a vertical $\mathrm{CT}$ measurement on $\mathrm{C}_{3-7}$ using $\mathrm{X}$-ray radiographs. However, the systematic reports concerning the vertical $\mathrm{CT}$ measurement of the thoracic pedicles and lumbar pedicles using CT scanning have not been available so far. In order to provide anatomical basis for the application of the transpedicular screw fixation, we conducted $\mathrm{CT}$ scanning of the bone matrix of vertebral pedicles $\left(C_{2}-L_{5}\right)$ in 30 intact spines and 24 patients.

\section{MATERIALS AND METHODS}

From 100 adult spinal specimens in the Inner Mongolia region, 15 male and 15 female spines were randomly selected and their the pedicle cortex and medullary cavity (WPC and WPMC), and the height of the pedicle cortex and medullary cavity (HPC and HPMC) as well as the bony cortex thickness of the medial, lateral, superior and inferior border at the pedicles isthmus of $C_{2}-L_{5}$ were measured respectively using CT scanning (Fig. 1).

\section{RESULTS}

\section{WPC, WPMC and WPMC/WPC}

Both WPC and WPMC of $C_{2}-L_{5}$ pedicles changed in a three-dovetailed-saddle shape (Table 1) with four peaks and three valleys, as follows: $C_{2}$ (high),
$\mathrm{C}_{4: 5}$ (low), $\mathrm{C}_{7}-\mathrm{T}_{1}$ (high), $\mathrm{T}_{4-5}$ (lowest), $\mathrm{T}_{11-12}$ (high), $L_{1}$ (low) and $L_{5}$ (highest). The ratio of WPMC/WPC changed with an obvious regularity, with all of the ratios above $55.0 \%$ and with the highest radio in $\mathrm{C}_{2}$ and the lowest ratio in $\mathrm{L}_{5}$. That indicated that medullary cavity of pedicles was comparatively larger than the bony cortex.

\section{WPC, WPMC and TPMC/TPLC}

The value of WPC minus WPMC was equivalent to the summed pedicle cortex thickness of medial border plus that of lateral border, the mean thickness being $2.1 \mathrm{~mm}$ among lumbar pedicles. The ratio of TPMC/ /TPLC changed from 3:1 to 2:1 and finally to $1: 1$ among cervical, thoracic and lumbar pedicles, respectively.

\section{HPC, HPMC and HPMC/HPC}

Both HPC and HPMC showed a saddle shape in the cervical segment, increasing gradually from $C_{5}$ to $L_{5}$ (Table 2), the lowest augment being from $C_{3}$ to $C_{6}$, the lowest value being in $\mathrm{C}_{5}$, and the highest value being in $T_{12}$ followed by $T_{11}$ and $L_{5}$. In terms of HPMC/HPC ratio, the value was the highest in $\mathrm{C}_{2}$, less than $50.0 \%$ in $\mathrm{C}_{3-6^{6}}$, between $57.0 \%$ and $66.7 \%$ in $\mathrm{C}_{7}-\mathrm{T}_{9}$, between $71.8 \%$ and $75.9 \%$ in $\mathrm{T}_{10}-\mathrm{L}_{5}$ with no obvious trend observed. That is to say, HPMC was $75.0 \%$ of HPC in $\mathrm{C}_{2}$ and $T_{10}-L_{5}$ while the ratio was less than $50.0 \%$ in $C_{3-6}$.

\section{HPC-HPWC and TPSC/TPIC}

The value of HPC minus HPMC is equivalent to the summed pedicle cortex thickness of superior border plus that of inferior border. The mean value of cervical pedicles was $3.3 \mathrm{~mm}$ in $\mathrm{C}_{3-6}$ but was lower in $\mathrm{C}_{2}$ and the mean value of both thoracic pedicles and lumbar pedicles was $4.0 \mathrm{~mm}$. The value of TPSC/TPIC changed mildly from $C_{2}-L_{5}$, ranging from 1.0 to 1.3 , which suggested that the thickness of the superior border was approximately equal to that of the inferior border (Table 2).

\section{WPMC/HPMC}

The WPMC/HPMC ratio of cervical pedicles was higher than $90.9 \%$, actually higher than $100.0 \%$ in $\mathrm{C}_{6-7}$ and lower than $50.0 \%$ in $\mathrm{T}_{4}-\mathrm{L}_{2}$, which suggested that WPMC of 11 pedicles in this region was less than half of HPMC (Table 2).

\section{DISCUSSION}

The spine is surrounded by trachea, oesophagus, thoracic aorta and heart, etc. Improper selection of screw length and diameter will not only cause injury 
Table 1. The width of the pedicle cortex and width of the pedicle medullary cavity (WPC and WPMC) and the mathematic relationship between them

\begin{tabular}{|c|c|c|c|c|c|}
\hline Vertebra & WPC & WPMC & WPMC/WPC & WPC-WPMC & TPMC/TPLC \\
\hline C2 & $7.6 \pm 1.8(4.3-12.5)$ & $6.0 \pm 1.4(4.0-7.8)$ & $78.9 \%$ & $1.6 \pm 0.4$ & 2.8 \\
\hline C3 & $5.1 \pm 0.7(3.5-6.8)$ & $3.1 \pm 0.7(2.0-4.3)$ & $60.8 \%$ & $2.0 \pm 0.4$ & 2.8 \\
\hline C4 & $5.0 \pm 0.8(3.3-6.8)$ & $3.0 \pm 0.8(1.8-4.6)$ & $60.0 \%$ & $2.0 \pm 0.5$ & 2.9 \\
\hline C5 & $5.2 \pm 0.8(3.5-6.9)$ & $3.0 \pm 0.6(1.9-4.4)$ & $55.7 \%$ & $2.2 \pm 0.4$ & 2.8 \\
\hline C6 & $5.6 \pm 0.9(3.5-7.8)$ & $3.3 \pm 0.6(1.9-4.4)$ & $58.9 \%$ & $2.3 \pm 0.5$ & 3.0 \\
\hline $\mathrm{C7}$ & $6.5 \pm 0.8(4.5-8.5)$ & $4.7 \pm 0.8(3.2-6.5)$ & $72.3 \%$ & $1.8 \pm 0.4$ & 3.2 \\
\hline $\mathrm{T} 1$ & $8.4 \pm 1.2(5.7-11.1)$ & $5.5 \pm 1.1(4.0-7.2)$ & $65.5 \%$ & $2.9 \pm 0.7$ & 1.9 \\
\hline $\mathrm{T} 2$ & $6.4 \pm 1.3(4.2-12.3)$ & $4.7 \pm 0.8(3.2-6.5)$ & $71.8 \%$ & $1.7 \pm 0.3$ & 2.0 \\
\hline T3 & $4.9 \pm 1.0(2.5-7.2)$ & $3.5 \pm 1.1(4.0-7.2)$ & $71.4 \%$ & $1.4 \pm 0.5$ & 2.5 \\
\hline T4 & $4.4 \pm 0.9(2.4-6.3)$ & $2.9 \pm 0.6(1.2-4.1)$ & $66.0 \%$ & $1.5 \pm 0.3$ & 2.1 \\
\hline T5 & $4.6 \pm 1.0(2.3-7.3)$ & $3.0 \pm 0.6(1.8-4.0)$ & $65.5 \%$ & $1.6 \pm 0.4$ & 2.2 \\
\hline T6 & $4.9 \pm 1.0(2.3-7.5)$ & $3.3 \pm 0.9(1.5-4.8)$ & $67.3 \%$ & $1.6 \pm 0.5$ & 2.0 \\
\hline $\mathrm{T} 7$ & $5.3 \pm 1.1(2.7-7.7)$ & $3.5 \pm 1.0(1.6-5.6)$ & $66.0 \%$ & $1.8 \pm 0.4$ & 2.3 \\
\hline T8 & $5.6 \pm 1.2(3.4-7.9)$ & $3.6 \pm 0.9(1.9-5.1)$ & $64.3 \%$ & $2.0 \pm 0.3$ & 2.6 \\
\hline T9 & $6.2 \pm 1.1(4.0-8.5)$ & $4.0 \pm 0.9(2.8-5.9)$ & $64.5 \%$ & $2.2 \pm 0.4$ & 2.2 \\
\hline $\mathrm{T} 10$ & $7.6 \pm 1.3(4.8-10.3)$ & $4.7 \pm 0.9(3.0-6.3)$ & $61.8 \%$ & $2.9 \pm 0.5$ & 2.3 \\
\hline T11 & $8.4 \pm 1.5(5.7-11.8)$ & $5.5 \pm 1.2(3.8-7.7)$ & $65.5 \%$ & $2.9 \pm 0.4$ & 2.2 \\
\hline $\mathrm{T} 12$ & $8.2 \pm 1.5(5.5-13.0)$ & $6.0 \pm 1.2(3.6-8.0)$ & $68.3 \%$ & $2.6 \pm 0.6$ & 2.0 \\
\hline L1 & $7.2 \pm 1.4(5.6-10.0)$ & $4.2 \pm 1.0(2.6-6.1)$ & $58.3 \%$ & $3.0 \pm 0.8$ & 1.3 \\
\hline L2 & $7.6 \pm 1.3(5.0-12.0)$ & $4.8 \pm 1.1(3.1-6.5)$ & $61.3 \%$ & $2.9 \pm 0.7$ & 1.2 \\
\hline L3 & $9.4 \pm 1.6(5.8-14.0)$ & $6.2 \pm 1.3(4.3-8.2)$ & $66.0 \%$ & $3.2 \pm 0.8$ & 1.1 \\
\hline L4 & $10.5 \pm 1.7(7.0-14.5)$ & $7.4 \pm 1.4(4.8-10.0)$ & $70.5 \%$ & $3.1 \pm 0.6$ & 1.2 \\
\hline L5 & $13.2 \pm 3.0(9.4-18.0)$ & $9.6 \pm 1.7(6.0-13.5)$ & $72.7 \%$ & $3.6 \pm 1.0$ & 1.1 \\
\hline
\end{tabular}

to the structure around the vertebra, but also damage the spinal cord, lead to paralysis of the patient. It is one of the critical factors in transpedicular screw fixation to choose the proper screw diameter, which is determined by pedicle morphology [4].

\section{Selection of screws}

In the lumbar segment WPMC is less than HPMC, so the screw diameter should be determined by WPMC. WPMC of $L_{3}$ and $L_{2}$ is less than half of HPMC, so the diameters of both screws should be determined by WPMC and two screws of $4.0-5.0 \mathrm{~mm}$ in diameter can be applied in $\mathrm{L}_{3-5}$. Only one screw with the corresponding diameter was applied in the surgeries involving the 16 cases of lumber injury and its effect was good, reducing risks of using two screws. Owing to the fact that the vertical $\mathrm{CT}$ image of the lumbar pedicles shows a vertical-ellipse shape similar to "O", the medullary cavity is large and the pedicle cortex of medical, lateral, superior and inferior borders is relatively thicker, great resistance was encountered during the placement of the threaded pin or screw. However, the safety of the surgical procedure was secured, which is one of the reasons why it is extensively applied in clinical practice. Some reports only presented the thickness of medial and lateral borders in $L_{3-5}[4]$, the value of which is comparatively lower than our measurement. Wu et al. [7] did not list the values of the lumbar pedicle cortex and its medullary cavity, but only their ratio, which is similar to our results, except for that of the $L_{1}$.

With only few exceptions (23.3\%) the WPMC of the thoracic pedicle is generally lower than HPMC in $T_{1}$, and the WPMC of $T_{4-5}$ is only about $3.0 \mathrm{~mm}$. Therefore, a pedicle hook can be applied in case of a screw that is too thin to use. The tomographs of the medullary cavity mostly show a round shape or a transverse-ellipse shape in $\mathrm{T}_{1}$, mostly a vertical-ellipse shape in $\mathrm{T}_{11}$ and $\mathrm{T}_{12}$, with the others being similar to an car shape or a sole shape with the upper part wider, the lower part narrower and the costal neck occupying the middle notch with extremely thin 
Table 2. The height of the pedicle cortex and height of the pedicle medullary cavity (HPC and HPMC) and the mathematic relationship between them

\begin{tabular}{|c|c|c|c|c|c|c|}
\hline Vertebra & HPC & HPMC & HPMC/HPC & HPC-HPMC & TPSC/TPLC & WPMC/HPMC \\
\hline $\mathrm{C} 2$ & $8.4 \pm 1.1(8.2-10.3)$ & $66.4 \pm 0.9(5.5-7.9)$ & $76.2 \%$ & $2.0 \pm 0.5$ & 1.2 & 93.7 \\
\hline C3 & $6.9 \pm 0.9(5.0-10.0)$ & $3.3 \pm 0.8(2.1-4.4)$ & $47.8 \%$ & $3.6 \pm 0.6$ & 1.1 & 93.9 \\
\hline C4 & $6.8 \pm 0.9(4.7-9.5)$ & $3.3 \pm 0.5(2.5-4.5)$ & $48.5 \%$ & $3.5 \pm 0.6$ & 1.1 & 90.9 \\
\hline C5 & $6.4 \pm 0.7(4.5-8.2)$ & $3.1 \pm 0.7(2.2-4.5)$ & $46.9 \%$ & $3.3 \pm 0.5$ & 1.1 & 96.7 \\
\hline C6 & $6.5 \pm 0.8(5.2-9.4)$ & $3.2 \pm 0.5(2.2-3.9)$ & $49.2 \%$ & $3.3 \pm 0.4$ & 1.1 & 103.1 \\
\hline C7 & $7.3 \pm 0.9(5.3-9.6)$ & $4.5 \pm 0.6(3.2-5.5)$ & $61.6 \%$ & $2.8 \pm 0.5$ & 1.0 & 104.4 \\
\hline T1 & $9.6 \pm 1.2(6.5-12.8)$ & $6.4 \pm 0.7(5.5-7.7)$ & $66.7 \%$ & $3.2 \pm 0.7$ & 1.2 & 85.9 \\
\hline T2 & $11.3 \pm 1.3(7.9-11.6)$ & $6.6 \pm 0.6(5.3-7.8)$ & $58.4 \%$ & $4.7 \pm 0.8$ & 1.2 & 71.2 \\
\hline T3 & $11.7 \pm 1.5(8.1-14.2)$ & $6.8 \pm 0.7(5.5-8.0)$ & $58.1 \%$ & $4.9 \pm 0.7$ & 1.3 & 51.5 \\
\hline T4 & $11.4 \pm 1.6(7.1-14.6)$ & $6.5 \pm 0.7(5.1-9.8)$ & $57.0 \%$ & $4.9 \pm 0.8$ & 1.3 & 44.6 \\
\hline T5 & $11.4 \pm 1.4(8.2-15.0)$ & $6.9 \pm 0.8(5.3-10.0)$ & $60.5 \%$ & $4.6 \pm 0.5$ & 1.1 & 43.5 \\
\hline T6 & $11.3 \pm 1.3(8.2-14.3)$ & $6.8 \pm 0.8(5.3-10.3)$ & $60.2 \%$ & $4.5 \pm 0.6$ & 1.1 & 48.5 \\
\hline $\mathrm{T7}$ & $11.5 \pm 1.3(8.3-14.3)$ & $7.1 \pm 0.8(5.5-10.2)$ & $61.7 \%$ & $4.4 \pm 0.5$ & 1.0 & 49.3 \\
\hline T8 & $12.0 \pm 1.3(8.8-14.2)$ & $7.3 \pm 0.9(5.8-10.6)$ & $60.8 \%$ & $4.7 \pm 0.7$ & 1.0 & 49.3 \\
\hline T9 & $12.9 \pm 1.4(9.6-15.4)$ & $8.5 \pm 0.8(6.1-11.8)$ & $65.9 \%$ & $4.4 \pm 0.6$ & 1.1 & 47.0 \\
\hline $\mathrm{T} 10$ & $14.9 \pm 1.5(10.8-18.2)$ & $10.7 \pm 1.1(8.3-14.0)$ & $71.8 \%$ & $4.2 \pm 0.7$ & 1.0 & 43.9 \\
\hline T11 & $16.6 \pm 1.6(12.4-20.5)$ & $12.6 \pm 1.2(9.9-14.4)$ & $75.9 \%$ & $4.0 \pm 0.6$ & 1.0 & 43.6 \\
\hline T12 & $16.9 \pm 1.5(14.1-22.5)$ & $12.8 \pm 1.3(9.8-15.2)$ & $75.7 \%$ & $4.1 \pm 0.7$ & 1.0 & 43.8 \\
\hline L1 & $15.5 \pm 1.4(10.2-18.8)$ & $11.4 \pm 1.2(8.2-14.0)$ & $73.5 \%$ & $4.1 \pm 0.6$ & 1.1 & 36.8 \\
\hline L2 & $15.0 \pm 1.5(11.0-18.1)$ & $11.1 \pm 1.1(8.5-13.5)$ & $74.0 \%$ & $3.9 \pm 0.5$ & 1.1 & 43.2 \\
\hline L3 & $14.6 \pm 1.5(10.3-18.2)$ & $10.7 \pm 1.3(8.3-13.0)$ & $73.3 \%$ & $3.9 \pm 0.5$ & 1.1 & 57.9 \\
\hline L4 & $14.5 \pm 1.9(9.6-19.8)$ & $10.5 \pm 1.4(8.0-13.3)$ & $72.4 \%$ & $4.0 \pm 0.8$ & 1.0 & 70.5 \\
\hline L5 & $16.7 \pm 2.8(11.3-25.0)$ & $12.4 \pm 2.0(8.6-15.9)$ & $74.3 \%$ & $4.3 \pm 0.6$ & 1.1 & 77.4 \\
\hline
\end{tabular}

pedicle cortex, which has no resistance to the screw. Therefore, the risks of placement into costovertebral joints and accidental injuries of the adjacent structures increase, which has great importance in clinical practice.

Few reports are concerned with the medullary cavity of cervical pedicle. Li et al. [3] reported that the screw diameter was determined by the WPMC after measuring WPMC and HPMC of $\mathrm{C}_{3-7}$ using X-ray photographs, which was similar to the results of our measurements, with a tolerable difference of less than $0.8 \mathrm{~mm}$. At the same time, Li et al. [3] reported that the value of $C_{5}$, which is the farthest from the gravitational line of human body, is the lowest, while WPMC is higher than HPMC in both $C_{6}$ and $C_{7}$. However, we believe that both WPMC and HPMC must be measured in $\mathrm{C}_{3-6}$, especially in $\mathrm{C}_{6}$ and $\mathrm{C}_{7}$, otherwise, pedicle fracture is likely to occur due to its narrow medullary cavity and the required accuracy in placement point, placement angle, diameter and length of the screw. The upper part of axis vertebral pedicle is obviously wider than either the middle or the lower part of it, but the pedicle cortex of the upper part is thicker than that of either the middle or the lower parts. As a result, the tomographs of the medullary cavity mostly manifest a vertical-ellipse shape or a round shape. It has been reported that screw fixation was not feasible for axis vertebra after the outer diameter of pedicles was measured [1], relevant research and clinical application studies have also been reported $[5,6]$. Screw fixation has been performed on a patient with fissure on the isthmus of the right side of $C_{2}$ pedicle into which a screw of $4.5 \mathrm{~mm}$ in diameter was successfully placed. The results of our study also show that the screw of $4.0-5.0 \mathrm{~mm}$ in diameter is feasible for $C_{2}$, but individual difference should be taken into consideration due to a large range of its WPMC and HPMC [6]. All of pedicle cortex of the lateral border in $\mathrm{C}_{2-7}$ is extremely thin while that of the superior, inferior and medial border is comparatively thicker, showing a typical C shape in CT image. Therefore, injuries of the cervical nerve root and/or vertebral 
vessels are likely to occur in case of screw deviating to the right and a vague resistance by hand sense.

The ideal diameter of the screw should be selected according to its corresponding medullary cavity. At the same time, some scholars claim that a slightly bigger screw should be used, which renders putting the screw into the pedicle cortex in order to strengthen its anti-extractability and anti-arcuatability [2]. In view of clinical experience, the nature of pedicle features and their critical adjacent structures, we believe that a slightly thinner screw is safer to use.

\section{Key points in clinical application}

According to anatomical observations and clinical documentations, tomographs of the $C_{2}$ pedicle indicating an ellipse shape of its medullary cavity, do not show its actual appearance and, therefore, the use of screw of $4.0-5.0 \mathrm{~mm}$ in diameter is feasible. The medullary cavity of $C_{3-6}$ is narrow, so CT scanning or magnetic resonance imaging scanning should be carefully performed to choose the screw of proper diameter. As far as $\mathrm{C}_{6}$ and $\mathrm{C}_{7}$ are concerned, diameter of the screw should be determined by HPMC or both HPMC and WPMC, the surgery should be monitored by $\mathrm{X}$-ray radiography for precise apposition and entry, and should be directed strictly in order to avoid injuries and serious complications.

The lateral bony cortex of cervical and thoracic pedicles is quite thin and offers hardly any resistance to the insertion of the screw, so great care should be taken during operation. In addition, the medullary cavity of $\mathrm{C}_{3-6}$ is narrow and so is that of $\mathrm{T}_{4-6}$, therefore, screw of $3.0 \mathrm{~mm}$ in diameter should be applied with caution, as such screw may be the only choice for the surgery.

\section{CONCLUSIONS}

Twenty-four patients were involved in the clinical application of the theory, including 17 males and 7 females aged 18-62 years, among whom one had spondyloschisis of $\mathrm{C}_{2}$ isthmus, two had $\mathrm{C}_{6-7}$ fracture, one had $T_{4-5}$ fracture, one had tumour of vertebral canal, one had $T_{5}$ fracture and the remaining patients had $T_{10}-L_{5}$ fractures. In all cases, the surgical procedures of the transpedicular screw fixation were performed, with the reference to the results of our measurements. All of the screws chosen for the surgeries were suitable except for one which was too thin and too loose for $T_{12}$. The therapeutic efficacy was generally satisfying.

\section{Financial support}

National science foundation of China (81460330;81560348,81260269); Inner Mongolia education bureau youth elite of science and technology (NJYT-15-B05); The Inner Mongolia autonomous region science and technology plan projects (2016); Science foundation of Inner Mongolia (2016MS08131); Inner Mongolia science and technology guidance project (2017).

\section{REFERENCES}

1. Cahueque Lemus MA, Cobar Bustamante AE, Ortiz Muciño A, et al. Clinical outcome of anterior vs posterior approach for cervical spondylotic myelopathy. J Orthop. 2016; 13(3): 123-126, doi: 10.1016/j.jor.2016.03.006, indexed in Pubmed: 27076742.

2. Koktekir E, Toktas ZO, Seker A, et al. Anterior transpedicular screw fixation of cervical spine: Is it safe? Morphological feasibility, technical properties, and accuracy of manual insertion. J Neurosurg Spine. 2015; 22(6): 596-604, doi: 10.3171/2014.10.SPINE14669, indexed in Pubmed: 25815805.

3. Li J, Zhao L, Qi F, et al. Three-dimensional finite-element study on anterior transpedicular screw fixation system of the subaxial cervical spine. Zhonghua Wai Ke Za Zhi. 2015; 53(11): 841-846, indexed in Pubmed: 26813839.

4. Ninomiya K, Iwatsuki K, Ohnishi Yl, et al. Radiological evaluation of the initial fixation between cortical bone trajectory and conventional pedicle screw technique for lumbar degenerative spondylolisthesis. Asian Spine J. 2016; 10(2): 251-257, doi: 10.4184/asj.2016.10.2.251, indexed in Pubmed: 27114765.

5. Srivastava SK, Nemade PS, Aggarwal RA, et al. Congenital absence of posterior elements of $C 2$ vertebra with atlanto-axial dislocation and basilar invagination: a case report and review of literature. Asian Spine J. 2016; 10(1): 170-175, doi: 10.4184/ asj.2016.10.1.170, indexed in Pubmed: 26949474.

6. Tang X, Cao Q, Chen L, et al. Anatomic study on entry point and implant technique for $\mathrm{C} 2$ pedicle screw fixation. hongguo Xiu Fu Chong Jian Wai Ke Za Zhi. 2015; 29(2): 175-178, indexed in Pubmed: 26455145.

7. Wu H, Chen Y, Zhang C, et al. [Application of percutaneous pedicle screw fixation for lumbar degenerative disease]. Zhonghua Yi Xue Za Zhi. 2014; 94(23): 1764-1768, doi: 10.3760/cma.j.issn.0376-2491.2014.23.002, indexed in Pubmed: 25154836. 